\title{
Conservation of Information-Processing Capacity in Paired-Associate Memorizing ${ }^{1}$
}

\author{
JAmes G. GReENo \\ University of Michigan, Ann Arbor, Michigan, 48104
}

\begin{abstract}
Data that impose constraints on hypotheses regarding the role of temporal variables in memorizing are reviewed, including results that apparently disconfirm Greeno's (1967) time-sharing hypothesis. An alternative hypothesis is proposed, in which it is assumed that $S$ occasionally attenuates his rate of processing information for memory, with the probability of attentuation being relatively high when the item being presented is still in short-term memory as a result of a recent presentation.
\end{abstract}

One of the best established, and least surprising, results in human verbal learning is that if we give $S$ s more time to study they usually learn more. An especially nice illustration of this was provided by Keller, Thomson, Tweedy, and Atkinson (1967), who gave

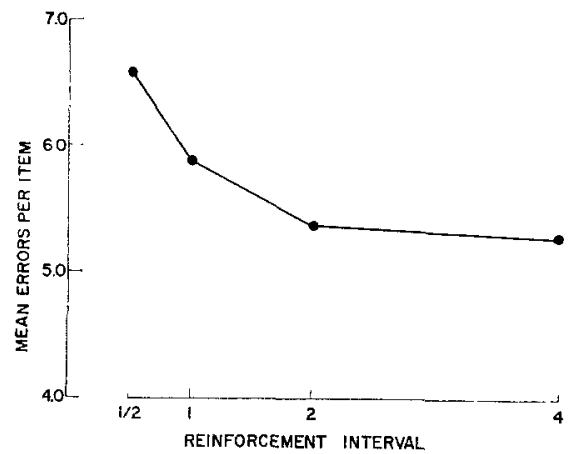

FIG. 1. Mean total errors in 15 trials as a function of duration of reinforcement interval. (After Keller et al., 1967.)

different amounts of study time on different number-letter paired associates in a mixed list. Their data are graphed in Figure 1, and the salient fact is the reduction of mean errors per item as study time is made greater.

These data emphasize the rather noncontroversial fact that in memorizing experiments,

\footnotetext{
1 This paper originated from the authors participation in the symposium, "Distribution Effects in Learning and Memory," Midwestern Psychological Association, May 9, 1969.
}

Ss process information in real time. While this fact, in itself, is nearly tautologous, interesting questions arise when we consider its implications for memorizing situations. An assumption of this paper is that processing for memory storage and retrieval involves activities carried out that affect items that are in $\bar{S}$ 's short-term memory. That is, when we give $S$ more time to study an item, we extend the time that he can process that item in short-term memory. It would be reasonable to assume that this extra time for processing permits either a longerlasting record of the item, or a more accessible record, or a better strategy for retrieving the item on test trials.

According to this idea, if an item were presented for study, and then immediately presented again, we should expect the second presentation to produce improved performance through its providing additional time for processing. This expectation is consistent with results obtained in two experiments conducted at Indiana University, one in which I collaborated with Susan White, and one with Donald Rumelhart. The experiments were basically comparisons among three conditions. In a condition called "normal," a list of items was presented in cycles of anticipation trials, with each item presented once during every cycle. A second condition was called "massed," and involved cycles in which each item was presented twice. In three-fourths of the cases, 
the two presentations of an item occurred in immediate succession, and in the remaining one-fourth of the cases an item's two presentations were separated by a single presentation of another item. In a third condition called "blank," the sequence was matched with the massed sequence, but during each interval containing the second presentation of an item in the massed condition, there was an interval for the blank condition in which no item was presented. In other words, during the intervals during which $S$ s in the massed condition saw items that they had seen very recently, $S$ s in the blank condition were given some free time to do whatever they wanted to do.

The list of items used in these experiments contained 15 paired associates, with familiar words as stimuli and the numbers $1-5$ as responses. The items were the same as those used in an earlier study (Greeno, 1964). The $S \mathrm{~s}$ in both experiments were students from introductory psychology courses at Indiana University who participated in order to fulfill class requirements.

In Mrs. White's experiment, items were printed on blank tabulating cards and presented using a modified key punch, with metal masks that allowed $S$ s to see only the stimulus on a test and the stimulus-response pair during study intervals. During a test interval, $S$ was instructed to press a button indicating his response. Each test interval lasted at least $2 \mathrm{sec}$, but the card did not advance until $S$ gave a response. In the blank condition when no item was presented, $S$ caused the card to advance by pressing a button marked "0." Each study interval was $2 \mathrm{sec}$ in duration. Between a study interval and the next test interval there was an interpresentation interval of either .6 or $1.0 \mathrm{sec}$, alternating between items. (Two items were printed on each card, and the difference in times was due to the time taken to change cards in the key punch.) Each $S$ was run to a criterion of two perfect cycles.

In Rumelhart's experiment, the items were presented to each $S$ on a television screen, with the image transmitted from a camera focussed on a computer printout of the items. Rumelhart ran $S$ s up to four at a time, and a test was terminated when all $S$ s responded. No response was required when no item was presented. The time allowed for study of each item was $1.5 \mathrm{sec}$, and an interval of $.5 \mathrm{sec}$ occurred between each presentation (test or study) and the next presentation. The $S$ s were run for 15 cycles of trials.

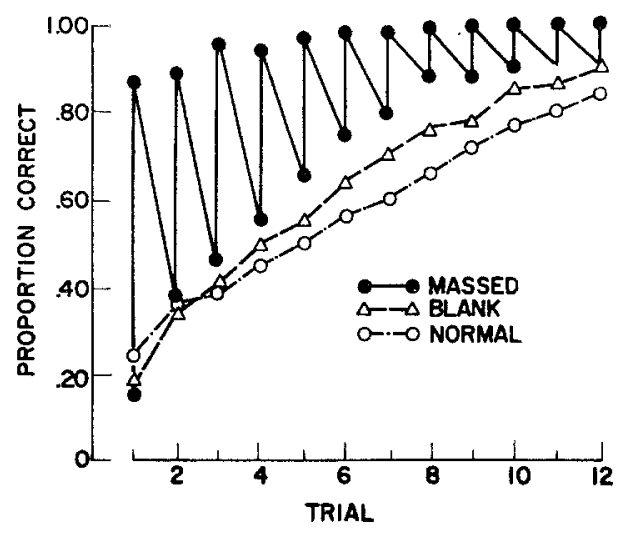

FIG. 2. Proportion correct response per trial, with massed items receiving two presentations per trial. (Data collected by Susan White.)

Mrs. White's results, from $20 \mathrm{Ss}$ per condition, are in Figure 2. In the learning curve for the massed condition each lower point is the performance on the first presentation of an item in a cycle, and each higher point is the performance on the second massed presentation. The groups with massed presentations and blank presentations made about the same mean number of errors- 4.62 and 4.94 per item, respectively-while more errors-6.17 per item-were made by the group with normal presentations.

Rumelhart's results are in Figure 3. In this case, the similarity between performance by the group with blank presentations and on the first presentations in the massed condition is apparent in the learning curves. The numbers of $S$ s were 12, 14, and 10, in the massed, blank, and normal conditions, respectively. The mean numbers of errors per item were 5.16, 4.80 , and 6.45 . 
It seems reasonable to infer from these data that the second massed presentation of an item is used as extra time by $S$, as we might have expected in the first place. However, the result was not entirely expected because of earlier

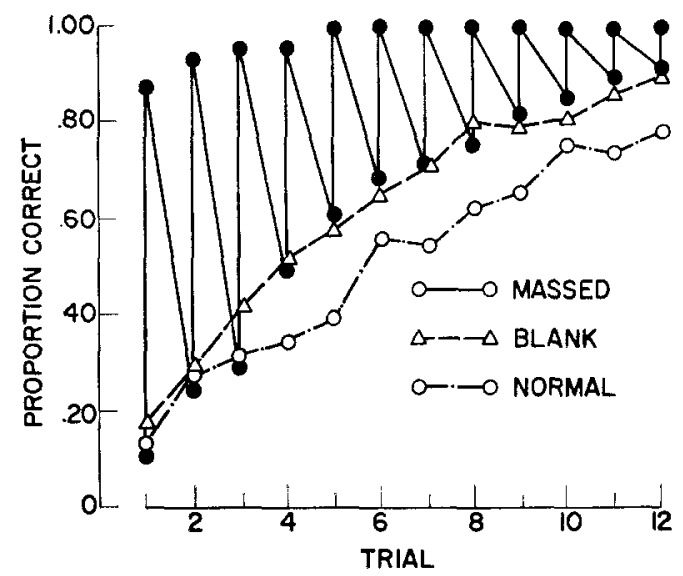

FIG. 3. Proportion correct responses per trial with massed items receiving two presentations per trial. (Data collected by Donald Rumelhart.)

findings to the contrary. In an earlier study (Greeno, 1964) there were single presentations and massed pairs of presentations mixed together in the same list. The results obtained in this mixed condition are in the left panel of Figure 4 . There is only a slight difference between performance on distributed items and on the first massed presentations. In the mixed-list experiment, the results suggest that two massed pairings are hardly more effective than a single presentation in the distributed condition. The difference between the two kinds of experiment is shown by comparing with the right panel of Figure 4, which averages the results from Figures 2 and 3 and omits the blank condition. There seems little doubt that the procedures produce different results.

The result obtained by Mrs. White and by Rumelhart seems easy to understand. The superiority of massed over single presentations in homogeneous lists seems like a simple result of giving $S$ s more time to study. On this view, the puzzling question is why the massed presentations in the mixed list are so ineffective. One possible answer to this question is a kind of time-sharing hypothesis (Greeno, 1967). According to this idea $S$ uses the time available during a massed presentation, but he does not necessarily use it for processing the item that is being presented. In most cases when a massed presentation occurs, there are other items in short-term memory along with the presented item. The time-sharing idea says that the time made available in the massed

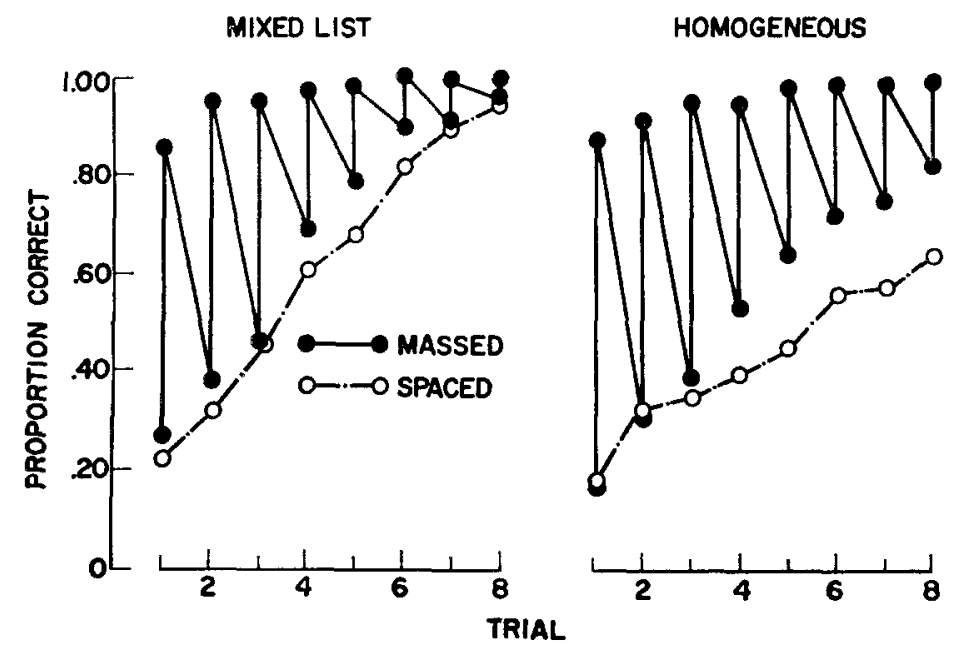

FIG. 4. Proportion correct responses per trial with massed and spaced presentations in a mixed list (from Greeno, 1964), and in separate homogeneous lists (average of White's and Rumelhart's data). 
presentation is shared between processing the item that is presented and the other item or items that are in short-term memory. In other words, this idea explains the failure of massed presentations to facilitate performance in a mixed list by the hypothesis that the extra time during a massed presentation is used to process not only the massed item, but also other items that are in short-term memory at that time.

While the time-sharing hypothesis is consistent with the data described above, there are other results that make the hypothesis seem dubious. One such kind of data has been collected by Potts (1969). Potts has compared the effectiveness of presentations that precede massed pairs of presentations on other items by different amounts. For example, in one study Potts had some items that always occurred immediately before a massed pair of presentations on a pair of items, or preceding a massed pair by one other item. A second set of items preceded a massed pair. of presentations by either two or three items. And a third set of items preceded a massed pair of presentations by four or five items. The hypothesis of time-sharing during massed repetitions implies that items that occur shortly before massed pairs of presentations should be learned faster than items further removed from massed presentations, since items closer to the massed presentations are more likely to be in short-term memory during the time that the extra time is given for processing. Potts has compared conditions of this general kind in several experiments, and has consistently found no difference among them.

A second kind of evidence has been obtained in studies of short-term retention of individual items. The time-sharing hypothesis attributes the ineffectiveness of massed repetitions to a choice by $S$ to process items other than the one that is presented. Peterson (1963) and Pollatsek (1969) have studied the spacing effect in a situation where this kind of sharing of processing cannot be effective. In these studies an item is presented, then $S$ counts backwards for an interval of time, then the item is presented again, there is another interval of counting backwards, and finally a retention test is given. The important comparisons involve items with different intervals between the two study presentations, with the interval between the second study presentation and the test held constant. If performance is better on items with longer intervals between study presentations, it cannot be due to sharing of processing time during the second study period. The reason is that $S$ has to retain only the single item that he is studying, and there would be no point in his going back to process earlier items. Nevertheless, a sizable spacing effect has been found, as is shown in Figure 5. An explanation based on total processing time is possible, but Pollatsek argued convincingly that this is inconsistent with the magnitude of the effect in his data, compared to the amount of retention that occurred after single presentations.

It should be recognized that the apparent inconsistency among these results may be due to procedural details that differed among the various experiments. For example, it could be that the duration of study presentations has an important effect on the effectiveness of a massed pairing of presentations. Another variable that could influence the results is the meaningfulness of the materials that $S$ is studying. Neither of these variables has been constant in the various experiments that I have discussed in this paper. However, on the present evidence it seems likely that an alternative to the time-sharing hypothesis should be considered.

The hypothesis that I now think is most likely is that $S$ sometimes simply turns off the processer that transfers items to long-term memory-or at least attenuates his rate of processing. According to this idea, $S$ simply does not process information during some conditions. And the probability of this happening is particularly high when the item being presented is already in $S$ 's short-term memory. 
This hypothesis is attractive only by elimination of alternatives; I am not aware of any direct evidence supporting it. However, consideration of the information-processing demands imposed in a memorizing task gives the idea a certain amount of plausibility. It seems reasonable to assume that the rate at which $S$ processes items for memory storage and retrieval must be variable, and at least partly under $S$ 's control. If $S$ concentrates carefully and expends great effort, he probably can memorize a relatively large number of items thing like most other biological activities, it should be possible to process information at very high rates for short periods relative to rates that can be sustained over longer periods of time.

The general assumption of this hypothesis is that in memorizing materials like paired associates, $S$ uses an information-processing system that is rate-limited. A further assumption is that with the usual procedures of rotelearning experiments materials are presented fast enough so that the rate at which items are

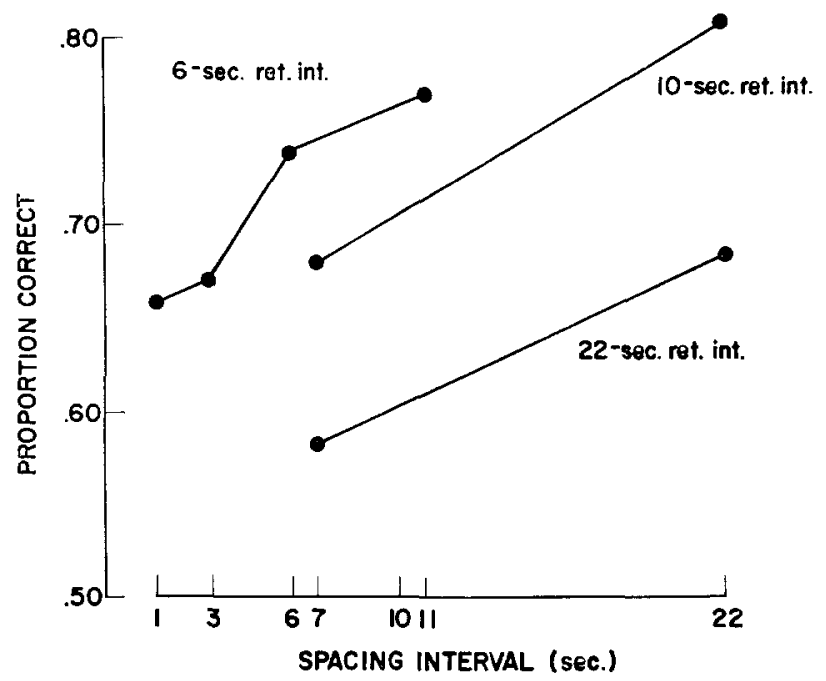

FIG. 5. Proportion correct reponses as a function of retention interval and spacing between two study presentations. (After Peterson, 1963, and Pollatsek, 1969.)

in a short time. But there is almost certainly an upper limit on the rate at which an individual can process items for memory.

In addition to the probable upper limit on the momentary rate of processing, it seems reasonable to suppose that there is another kind of limit involving processing capacity over extended intervals of time. First, consider a possibility that almost certainly gives too high an upper limit: the maximum rate of processing information for memory multiplied by the duration of the time interval. It seems virtually certain that $S$ 's capacity for memorizing items must be lower than this quantity. If information-processing for memory is any- presented exceeds the rate at which $S$ can process them for retention. In such a situation, $S$ can capitalize on his ability to control his rate of processing. If $S$ occasionally takes time out from processing items for a short time, he probably can operate at a higher rate during the time that he is processing items.

These considerations suggest that there are times during a memorizing task when it is efficient for $S$ to reduce his rate of processing items. If this is granted, then it seems reasonable to expect that the probability of attenuating the rate of processing should be relatively high when the presented item is one that has been processed recently - especially if the item 
is still in short-term memory when it is shown. This hypothesis, while rather inelegant, does allow for the variety of effects of massed and distributed presentations that I have described in this paper. It permits spacing effects in situations like those found with mixed lists (Greeno, 1964) and with single items (Peterson, 1963; Pollatsek, 1969) without requiring retroactive facilitation so that it is consistent with Potts' (1969) data. And it permits an overall facilitation of memorizing when massed presentations are given in a homogeneous list in accord with White's and Rumelhart's data, accounting for this effect on the basis of an increase in the time available to $S$ for processing items.

\section{REFERENCES}

Greeno, J. G. Paired-associate learning with massed and distributed repetitions of items. Journal of Experimental Psychology, 1964, 67, 286-295.
Greeno, J. G. Paired-associate learning with short term retention: mathematical analysis and data regarding identification of parameters. Journal of Mathematical Psychology, 1967, 4, 430-472.

Keller, L., Thomson, W. J., Tweedy, J. R., \& AtKiNsoN, R. C. Effects of reinforcement intervals in paired-associate learning. Journal of Experimental Psychology, 1967, 73, 268-277.

PETERSON, L. R. Immediate memory: data and theory. In C. N. Cofer and B. S. Musgrave (Eds.), Verbal behavior and learning. New York: McGrawHill, 1963. Pp. 336-353.

PollatseK, A. W. Rehearsal, interference, and spacing of practice in short-term memory. Unpublished doctoral dissertation, The University of Michigan, 1969.

PotTS, G. R. Distance from a massed double-presentation as a factor in paired-associate learning. Paper read at meetings of the Midwestern Psychological Association, Chicago, May, 1969.

(Received April 3, 1970) 